\title{
Spin polarization in the photoemission from the $5 d$-core levels of a thallium film
}

\author{
B. Schmiedeskamp, B. Vogt, U. Heinzmann
}

Fakultät für Physik, Universität Bielefeld, W-4800 Bielefeld 1, Federal Republic of Germany Fritz-Haber-Institut der Max-Planck-Gesellschaft, W-1000 Berlin 33, Federal Republic of Germany

Received: 15 September 1992

\begin{abstract}
A thick unordered T1 film has been deposited on $\mathrm{Pt}(111)$. Photoemission with photon energies between $20 \mathrm{eV}$ and $27 \mathrm{eV}$ reveals two peaks due to emission from the $5 d_{3 / 2^{-}}$and the $5 d_{5 / 2^{-}}$levels of $\mathrm{Tl}$. The measurements are performed with circularly polarized radiation. Spin analysis allows separation of the photoelectron intensity $I$ into its parts $I_{+}$with spin polarization vector parallel and $I_{-}$antiparallel to the photonspin. For the $d_{3 / 2^{-}}$and the $d_{5 / 2}$-level the ratio $\frac{I_{+}}{I_{-}}$turns out to be approximately $3: 1$ and 1:1.8 respectively. These values are close to the values expected for $d$-level photoionization of free atoms if spin orbit interaction in the final states is neglected. A slight crystal field splitting of $0.2 \mathrm{eV}$ is observed for the $d_{5 / 2}$-level. The data are compared with results for the photoemission from $\mathrm{Pb}$ films [1] and data for the photoionization from the $d$-levels of $\mathrm{Hg}$ [2].
\end{abstract}

\section{Introduction}

Spin-, energy- and angle-resolved photoemission with circularly polarized radiation has in the past been applied to a number of non-magnetic single-crystalline surfaces and adsorbates [3]. Symmetries of electronic states and bands could be determined and information is especially gained about interactions between adsorbate atoms and their surroundings [3-6]. The information can most easily be deduced from spin resolved photoemission data, if the photoemission can be described as being due to transitions from only two $m_{j}$-sublevels of the adsorbate state and if it yields completely spin polarized electrons for each of the two $m_{j}$-sublevels with opposite spin polarization signs.

The size of the crystal field splitting can then be precisely determined and the $m_{j}$-sublevels can be identified by the spin polarization sign. These conditions are approximately fulfilled if spin orbit interaction of the final states can be neglected and if the photoemission is performed for the highly symmetrical experimental setup of normal light incidence and normal electron emission. In a number of examples the technique has been applied earlier to the weakly bound rare gas Xe $[3-5,7]$.

The spin orbit splitting of the $p$-hole-state is in this case larger than crystal field splittings and many details of the photoemission process can be described in analogy to free Xe-atom photoionization [3-5, 7]. For a Xe atom adsorbed in the dilute phase on $\mathrm{Pd}(111)$, for example, the crystal field splitting vanishes and spin polarization values of $100 \%$ and $-50 \%$ are obtained [5] for the $p_{\frac{1}{2}}$ and the $p_{\frac{3}{2}}$-hole state, respectively, in agreement with values for free Xe-atom photoionization, if spin orbit interaction of the final states can be neglected.

Several studies have been reported about spin resolved photoemission from $d$-bands of metals and metal adsorbates $[3,8,9]$ but in most cases the crystal field splitting due to metal metal interaction is larger than the spin orbit splitting. For this reason and due to the fact that the $d$-core levels of most materials (which have of course smaller $d$-electron overlap and larger spin orbit splitting) are not accessible with most existing sources of circularly polarized light, no example has been reported up to now in which the spin polarization of photoelectrons from $d$ levels of an adsorbate system approach quantitatively the same values as for the $d$-level photoionization of a free atom for which spin orbit interaction of the final states can be neglected. Such an example is given in this letter.

An earlier attempt to achieve such an example, $\mathrm{Pb}$ on $\mathrm{Pt}(111)$, is described in [1]. As for $\mathrm{Tl} / \mathrm{Pt}(111)$ the spin orbit splitting of the $5 d$ core levels was considerably larger than crystal field splittings but the spin polarization values for lead on $\mathrm{Pt}(111)$ turned out to be considerably smaller than the values for $\mathrm{Tl}$ in this work. Possible reasons for this difference are discussed. The photoionization process for the $5 d$-levels of $\mathrm{Hg}$ has been studied in [2] and compared with relativistic and non-relativistic calculations. For photon energies slightly above the threshold for photoionization considerable discrepancies are found between experiment and both relativistic and non-relativistic calculations, while at higher photon 
energies the three sets of data are almost identical. Close to the threshold there is a remarkable influence of the spin orbit interaction in the final electronic states due to a pronounced variation of the matrix elements and thus cross sections and branching ratios (shape resonance).

\section{Experimental}

The experiments were done with circularly polarized radiation at the $6.5 \mathrm{~m}$ normal incidence monochromator [10] at BESSY. The experimental setup is described in [11]. The photoemission was performed for the highly symmetrical experimental setup of normal light incidence and normal electron emission from the $\mathrm{Tl}$ adsorbate layer on $\mathrm{Pt}(111)$. The photoelectron energy is determined with a simulated hemispherical electron spectrometer. The overall energetic resolution (electrons plus photons) for the present study was better than $200 \mathrm{meV}$ at an angular resolution of $\pm 3^{\circ}$.

The $\mathrm{Pt}(111)$ crystal was mounted on top of a manipulator. Its surface normal coincided within $0.5^{\circ}$ with the (111)-direction and within $0.3^{\circ}$ with the propagation direction of the light. A clean $\mathrm{Pt}(111)$ surface was obtained by applying $\mathrm{Ar}^{+}-$and $\mathrm{Ne}^{+}$bombardment, heating in oxygen and flashing. The procedure was controlled by Auger electron spectroscopy (AES) and LEED.

Thallium was deposited onto the surface at room temperature by means of a resistively heated evaporator [1] surrounded by a cooled shield and placed at a distance of about $20 \mathrm{~cm}$ from the Pt crystal. The thallium beam between evaporator and Pt crystal was almost completely enclosed by a tube which has only an orifice directly in front of the Pt crystal. After initial outgasing the evaporator worked without strong influence on the UHV-conditions. The deposition was always done for short periods, in between the surface was controlled by LEED and Auger.

\section{Results and discussion}

As in an earlier study of $\mathrm{Pb}$ on $\mathrm{Pt}(111)$ [1] the growth was studied by AES and LEED and yielded also a Stranski-Krastanov growth. Since the photoemission intensities were low and the secondary electron background was high for $\mathrm{Tl}$ coverages in the monolayer range the photoemission studies with spin analysis were performed for a thick Tl layer on Pt (about 10 layers). For this coverage only a diffuse LEED pattern was observed and no Auger signal from the underlying $\mathrm{Pt}$ surface. Photoemission intensities were measured for photon energies between 20 and $27 \mathrm{eV}$. They are given in Fig. 1 and all show peaks at $12.5 \mathrm{eV}$ and $14.8 \mathrm{eV}$ below $E_{F}$ due to photoemission from the $5 d_{5 / 2}$ and $5 d_{3 / 2}$ core level of Tl.

Spin analysis results in the spectra of Fig. 2. In the upper part the total intensity $I$ is given. By means of the measured spin polarization $P$ this intensity is separated into the partial intensities $I_{+}=\frac{I}{2}(1+P)$ and

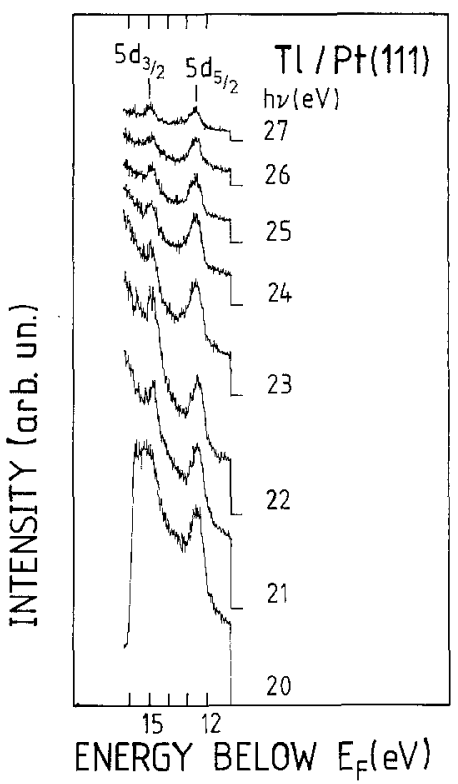

Fig. 1. Intensities for the normal photoemission from the $d$-core levels of $\mathrm{Tl}$ in a $\mathrm{Tl}$ film on $\mathrm{Pt}(111)$

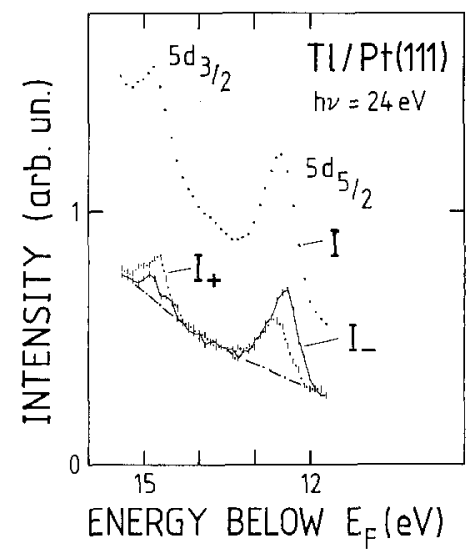

Fig. 2. Spin resolved photoemission from a $\mathrm{Tl}$ film on $\mathrm{Pt}(111)$ with circularly polarized radiation. $I_{+}$and $I_{-}$denote the intensities with spin polarization vector parallel and antiparallel to the photon spin. Error bars include the statistical error of the count rates and the uncertainties of the light polarization and the detector asymmetry function. Dash-dotted lines below the peaks indicate the secondary electron background

$I_{-}=\frac{I}{2}(1-P)$ with spin parallel and antiparallel to the photon spin. For the $5 d_{5 / 2}$-level $I_{-}$is considerably larger than $I_{+}$, for the $5 d_{3 / 2}$ level $I_{+}$turns out to be larger than $I_{-}$. In order to arrive at the true photoelectron intensity from the Tl-adsorbate we have to subtract a background of secondary electrons. With the background subtraction indicated by the dash-dotted lines below the peaks we result at intensity ratios $I_{+} / I_{-}$of $3: 1$ and $1: 1.8$ for the $5 d_{3 / 2^{-}}$and $5 d_{5 / 2}$-level, respectively, with an uncertainty of about $5 \%$ mainly due to uncertainties in the background subtraction.

These values shall be compared with values expected for the free atom $d$-level photoionization. Figure 3 shows the corresponding level scheme. It is based on the as- 


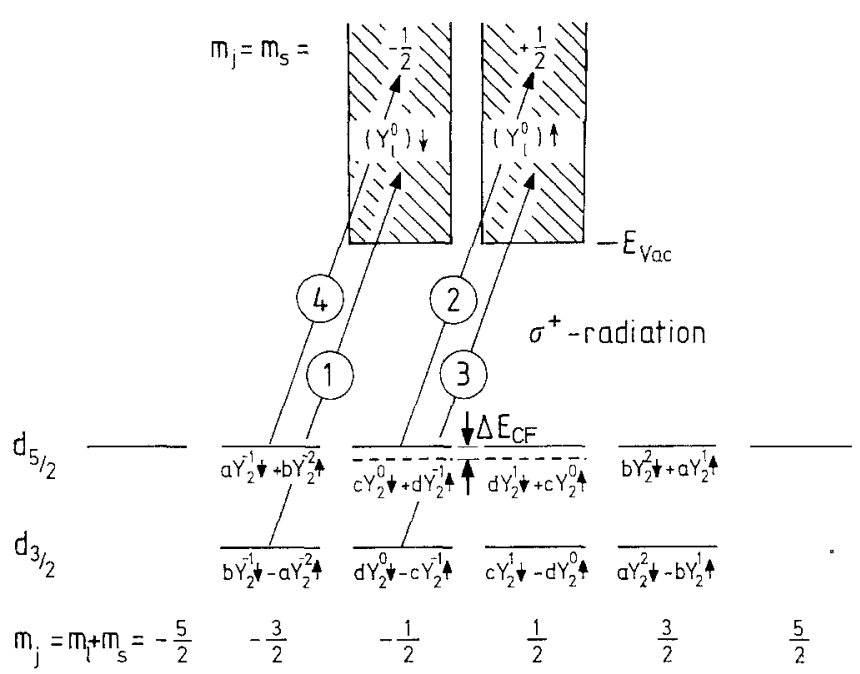

Fig. 3. Level scheme for the photoionization from the $d$-levels of a free atom with incident $\sigma^{+}$-radiation and alignment with the photoelectron emission direction. Solid lines represent initial states, the hatched areas final states and the arrows with encircled numbers the dipole allowed transitions with relative transition probabilities. Dashed lines show how the $d_{5 / 2}$-levels are changed by a small crystal field splitting $\Delta E_{\mathrm{CF}}$. $Y_{l}^{m_{i}}$ 's with prefactors are the spherical harmonics with Clebsch Gordon coefficients, small arrows represent the spinors. Final states are pure spin states, which consist of linear combinations of $Y_{I}^{m}$ 's with $m_{l}=0$ only

sumption that spin orbit interaction of the final states can be neglected and that the radial parts of the wave functions are not affected by spin orbit interaction [12]. The $d_{5 / 2}$ and $d_{3 / 2}$-levels are 6- and 4-fold degenerate, respectively. Since only the photoelectrons with emission direction antiparallel to the light propagation direction are studied, $m_{l}=0$ and thus $m_{j}=m_{s} \pm 1 / 2$ is required for the final states. This restricts the dipole allowed transitions for $\sigma^{+}$-radiation to only two transitions for each of the two $d$-levels. The two transitions yield completely spin polarized photo electrons with opposite signs. The relative transition probabilities for the two transitions are only determined by the squares of the Clebsch Gordon coefficients $a=\frac{2}{\sqrt{5}}, b=\frac{1}{\sqrt{5}}, c=\frac{\sqrt{3}}{\sqrt{5}}$ and $d=\frac{\sqrt{2}}{\sqrt{5}}$ and result in a relative weight of $c^{2}: b^{2}=3: 1$ for photoemission from the $m_{j}=-\frac{1}{2}$ and $m_{j}=-\frac{3}{2}$ sublevels of the $d_{3 / 2}$ state. Within this model the intensities $I_{+}$and $I_{-}$in the $d_{3 / 2}$-peak of Fig. 2 can also directly be correlated with the two transitions from the $d_{3 / 2}$-levels in the level scheme of Fig. 3. In the same way the intensities $I_{+}$and $I_{-}$in the $d_{5 / 2}$-peak of Fig. 2 are due to the two transitions from the $m_{j}$-sublevels of the $d_{5 / 2}$-state given in Fig. 3. In this case the ratio of the transition probabilities is $d^{2}: a^{2}=2: 4$ in fair agreement with the experimental value of $1: 1.8$. Both ratios are also identical with the theoretical prediction of Cherepkov [13] in the so-called "non relativistic" approximation (no spin orbit interaction in the final states). In this approximation the spin polarization value is only determined by the Clebsch Gordon coefficients and not by matrix elements and phase shift differences.
The crystal field splitting $\Delta E_{C F}$ has been determined from the centres of gravity of the $I_{-}$and $I_{+}$-parts of the $d_{5 / 2}$ peak in Fig. 2 . It is represented by means of the dashed lines in the level scheme of Fig. 3, turns out to be about $0.2 \mathrm{eV}$ and is thus almost identical with the value determined for $\mathrm{Pb}$ films in [1]. For the $d_{3 / 2}$ peak it is smaller than the experimental resolution of $0.1 \mathrm{eV}$.

The spin polarization for the same photon energy of $24 \mathrm{eV}$, however, was for the $d_{5 / 2}$-level of $\mathrm{Pb}$ by a factor of $4\left(I_{+}: I_{-} \approx 1: 1.2\right)$ smaller than for $\mathrm{Tl}$ in this work.

In order to discuss possible reasons for this discrepancy we compare the results for Tl films and $\mathrm{Pb}$ films with data for the $d$-photoionization of $\mathrm{Hg}$, which has the same electronic configuration of the cores as $\mathrm{Tl}$ and $\mathrm{Pb}$. For $\mathrm{Pb}$ films $h v=24 \mathrm{eV}$ is a photon energy slightly above the threshold of photoemission, while for Tl the same photon energy is more than $5 \mathrm{eV}$ above the threshold. In the photoionization experiment for $\mathrm{Hg}$ [2] the spin polarization is also almost zero [14] for photon energies slightly above threshold and approaches the values expected from the Clebsch Gordon coefficients according to the model in Fig. 3, if the photon energy is more than $5 \mathrm{eV}$ above threshold. This behaviour of $\mathrm{Hg}$ is explained by a breakdown of the non-relativistic approximation within the threshold region where the cross section and thus the matrix elements show a pronounced shape resonance structure due to the centrifugal barrier which results in non-statistical distributions of matrix elements for transitions into $p_{3 / 2^{-}}, p_{1 / 2^{-}}, f_{7 / 2^{-}}, f_{5 / 2^{-}}$waves. An additional hint that this mechanism might also be responsible for the low spin polarization of $\mathrm{Pb}$ films in the threshold region is given by the photoelectron intensities. As in $\mathrm{Hg}$ [2], in the photoionization of $\mathrm{Pb}$ [16] one observes a strong intensity variation with photon energy in the threshold region due to a shape resonance. An increase of intensity with photon energy is also observed in the threshold region for the photoemission from Pb-films (Fig. 1 in [1]).

\section{Conclusion}

We have studied the spin resolved photoemission with circularly polarized radiation of a $\mathrm{Tl}$ film on $\mathrm{Pt}(111)$. This system turns out to be the first example, in which the spin polarization of the photoelectrons emitted from $d$-levels approaches quantitatively the spin polarization values expected for free atom photoionization with neglection of spin orbit interaction in the final states. Earlier experiments with $\mathrm{Pb}$-films and free $\mathrm{Hg}$ atoms are discussed in order to point out why the spin polarization of photoelectrons is considerably lower for these samples and photon energies close to the threshold of photoemission.

We express our thanks to P. Stoppmanns and to the BESSY staff for their assistance. The work was financially supported by the BMFT (05431 AXB). 


\section{References}

1. Vogt, B., Schmiedeskamp, B., Heinzmann, U.: Vacuum 41, 1118 (1990)

2. Schäfers, F., Heckenkamp, Ch., Schönhense, G., Heinzmann, U.: J. Phys. B21, 769 (1988); Schäfers, F., Heckenkamp, Ch., Müller, M., Radojević, V., Heinzmann, U.: Phys. Rev. A42, $2603(1990)$

3. Heinzmann, U.: Phys. Scr. T17, 77 (1987); In: Campagna, M., Rosei, R. (eds.): Photoemission and absorption spectroscopy of solids and interfaces with synchrotron radiation, pp. 469. Amsterdam: North Holland 1990

4. Heinzmann, U., Schönhense, G.: Chap. 11, In: Feder, R. (ed.): Polarized electrons in surface physics. Singapore: World Scientific 1985. Schönhense, G.: Appl. Phys. A41, 39 (1986)

5. Vogt, B., Kessler, B., Müller, N., Schönhense, G., Schmiedeskamp, B., Heinzmann, U.: Phys. Rev. Lett. 67, 1318 (1991)

6. Vogt, B., Schmiedeskamp, B., Heinzmann, U.: Phys. Rev. B42, $9267(1990)$

7. Kessler, B., Müller, N., Schmiedeskamp, B., Vogt, B., Heinzmann, U.: Z. Phys. D17, 11 (1990)
8. Schmiedeskamp, B., Kessler, B., Vogt, B., Heinzmann, U.: Surf. Sci. 223, 465 (1989)

9. Vogt, B., Schmiedeskamp, B., Heinzmann, U.: Z. Phys. B80, 359 (1990)

10. Schäfers, F., Peatman, W., Eyers, A., Heckenkamp, Ch., Schönhense, G., Heinzmann, U.: Rev. Sci. Instrum. 57, 1032 (1986)

11. Eyers, A., Schäfers, F., Schönhense, G., Heinzmann, U., Oepen, H.P., Hünlich, K., Kirschner, J., Borstel, G.: Phys. Rev. Lett. 52, 1559 (1984)

12. Meier, F., Pescia, D.: Chap. 7. In: Meier, F., Zakharchenya, B.P. (eds.): Optical orientation. Amsterdam: Elsevier 1984

13. Cherepkov, N.A.: Adv. At. Mol. Phys. 19, 395 (1983)

14. The spin polarization values can be determined by means of the equation $P=\frac{A-\alpha}{1-\beta / 2} . A$ - and $\alpha$-values are displayed in Fig. 3 of [2], $\beta$-values are given in [15]

15. Schönhense, G., Schäfers, F., Heinzmann, U., Kessler, J.: Z. Phys. A304, 31 (1982)

16. Sandner, N., Schmidt, V., Mehlhorn, W., Wuilleumier, F., Adam, M.Y., Desclaux, J.P.: J. Phys. B13, 2937 (1980) 